Vietnam Journal of Mechanics, VAST, Vol. 34, No.2 (2012), pp. $101-112$

\title{
EVALUATION OF INTERFACIAL TOUGHNESS FUNCTION IN MIXED MODE LOADING
}

\author{
Do Van Truong, Vuong Van Thanh \\ Hanoi University of Science and Technology, Vietnam
}

\begin{abstract}
Interfacial strength is one of important factors to affect working stability of structures and devices. In order to avoid interfacial cracking, an improved method based on the conventional one is proposed to establish the interfacial toughness function (or the set of the interfacial fracture criteria in mixed-mode loading). By preliminarily analyzing the Brazil nut specimen, the placements of applied load, where pure modes I and II occurred, were determined. Experiment was then conducted on the specimens at the pre-determined placements of applied load. The interfacial toughness function was finally established by an empirical function based on only the critical energy release rates at pure modes I and II. The results showed that the interfacial toughness function was close to that obtained by experimental data and other researches.
\end{abstract}

Key words: Interface toughness, fracture criteria, interface crack, bi - material.

\section{INTRODUCTION}

Adhesively bonded joint has been employed in many industries, especially in micro/nano electronic and aerospace industries, due to its advantages such as small, light structure and use of fewer details. The joint can be created by two main methods as the indirect method and the direct method depending on its application and size scale. For material layers in supermicron-scale, the joint is usually formed from two surfaces adhered together by a bonding agent as heat and pressure, while the joint of material layers in submicron-scale is directly formed by evaporating or sputtering the atoms of a source material on the surface of a substrate material. The adhesion strength of formed interface depends on component material layers, surface quality and joint angle between material layers as well as fabrication method. Since the reliability of adhesion between material layers is one of important factors to affect working stability of structures and devices, the evaluation of interfacial fracture criteria between material layers is a necessary task.

Many methods have been proposed to evaluate the interfacial fracture criteria such as the peeling test [1,2], the four-point bending test [3], the sandwiched cantilever test [4] and the double cantilever beam test [5]. However, the mentioned methods can not control the mix of loading, and only can calibrate the interfacial fracture criteria at pure mode I and some combinations of mode I and mode II. Recently, the Brazil nut specimen test [6] and the Arcan specimen test [7] have been popular used in the interfacial cracking experiment to evaluate the interfacial toughness function (or the interfacial toughness 
curve). These methods can calibrate the interfacial toughness not only at pure mode I and pure mode II but also at almost any combination of mode I and mode II. Although the interfacial toughness function is established by the methods, the function obtained was a complicated, a non-general form and is difficult for use. In addition, the preparation of many Brazil nut specimens with a pre-crack in experiment meets difficulties, especially for the specimens with material layers in submicron-scale.

In this study, an improved method based on the conventional one is proposed to establish the interfacial toughness function. A key additional step in the improved method was to preliminarily analyze the Brazil nut specimen by FEM to determine the placements of applied load, where pure modes I and II occurred. Experiment was then conducted on the specimens at the pre-determined placements of applied load. The interfacial toughness function was finally established by an empirical function based on only the energy release rates in pure mode I toughness $G_{\mathrm{I}}^{c}$ and pure mode II toughness $G_{\mathrm{II}}^{c}$. Moreover, the mixedmode interfacial fracture criteria were reviewed, and the parameters such as the second Dundurs' parameter $\beta$ affecting the criteria were considered as well.

\section{MIXED-MODE INTERFACIAL FRACTURE CRITERIA}

Fig. 1 shows the general configuration near the crack tip on the interface bonded by two dissimilar isotropic materials. Material 1 with the subscript 1 is above the interface

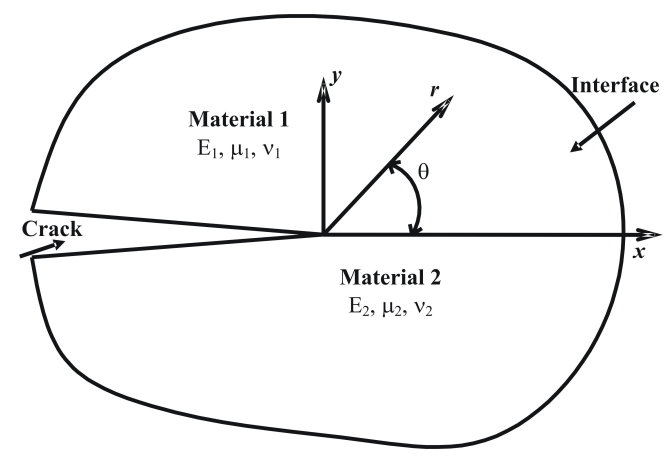

Fig. 1. Bi-material with a pre-crack.

and material 2 with the subscript 2 is below. $E_{i}, \nu_{i}$ and $\mu_{i}(i=1,2)$ are the Young's modulus, the Poisson's ratios and the shear modulus of the respective materials. For the crack tip as shown in the figure, the singular stress field ahead of the crack tip under small-scale yielding condition is expressed by the following equation

$$
\sigma_{22}+i \sigma_{12}=\left(K_{\mathrm{I}}+i K_{\mathrm{II}}\right)(2 \pi r)^{-1 / 2} r^{i \varepsilon}
$$

with

$$
r^{i \varepsilon}=\cos (\varepsilon \ln r)+i \sin (\varepsilon \ln r) .
$$

Here, $K_{I}$ and $K_{I I}$ are the interfacial stress intensity factors which play similar roles to conventional mode I and mode II intensity factors, $r$ is the distance from the tip, $i$ is the 
imaginary number and $\varepsilon$ is the oscillation index given by

$$
\varepsilon=\frac{1}{2 \pi} \ln \left(\frac{1-\beta}{1+\beta}\right)
$$

where $\beta$ is the second Dundurs' parameter measuring the mismatch in the in-plane bulk modulus and is defined by

$$
\beta=\frac{1}{2} \frac{\mu_{1}\left(1-2 \nu_{2}\right)-\mu_{2}\left(1-2 \nu_{1}\right)}{\mu_{1}\left(1-\nu_{2}\right)+\mu_{2}\left(1-\nu_{1}\right)}
$$

As in elastic fracture mechanics for homogeneous isotropic solid, two parameters such as the energy release rate and the interfacial stress intensity factor are also used to describe the behavior of cracks in mechanics of interface. The former quantifies the change in potential energy accompanying an increment of crack extension; the later characterizes the stress, strain and displacement near the crack tip. The energy release rate $G$ describes global behavior while the interface stress intensity factor $K$ is a local parameter. For linear elastic bi-material, a relationship between $G$ and $K$ is given as [8]

$$
G=\frac{\left(1-\beta^{2}\right)}{E_{*}}\left(K_{\mathrm{I}}^{2}+\lambda K_{\mathrm{II}}^{2}\right)
$$

with

$$
E_{*}=\frac{2 E_{1}^{\prime} E_{2}^{\prime}}{\left(E_{1}^{\prime}+E_{2}^{\prime}\right)}
$$

where $E_{i}^{\prime}$ is equal to $E_{i}$ in plane stress condition and $E_{i}^{\prime}$ is equal to $E_{i} /\left(1-\nu_{i}^{2}\right)$ in plane strain condition. $\lambda$ is a constant considering the influence of mode II contribution in the criterion. The magnitude of $\lambda$ varies between 0 and 1 and has to be determined from experiment. The interface between material layers is considered as a brittle one when $\lambda$ closes to 1 .

The interfacial strength is characterized by the energy release rate $G$ and the interface stress intensity factor $K$. The crack initiates to propagate along the interface when either the energy release rate or the interface stress intensity factor reaches the critical values. The interface toughness of interface crack is known as these critical values. From Eq. (5), the mixed-mode fracture criterion for an interface crack relating to the stress intensity factors can be given as

$$
\left(\frac{K_{\mathrm{I}}}{K_{\mathrm{I} c}}\right)^{2}+\left(\frac{K_{\mathrm{II}}}{K_{\mathrm{II}}}\right)^{2}=1
$$

with

$$
K_{\mathrm{I}_{c}}=\sqrt{\frac{G_{\mathrm{I}}^{c} E_{*}}{\left(1-\beta^{2}\right)}}, \quad K_{\mathrm{II}_{c}}=\sqrt{\frac{G_{\mathrm{I}}^{c} E_{*}}{\lambda\left(1-\beta^{2}\right)}}
$$

where the subscript $c$ refers to the critical values of the stress intensity factors and $G_{\mathrm{I}}^{c}$ is the energy release rate in pure mode I toughness. Fig. 2 depicts this mixed-mode interfacial fracture criterion under an elliptical law with two semi-axis of $K_{\mathrm{I}_{c}}$ and $K_{\mathrm{II}_{c}}$. Pure mode I occurs when $K_{I I}$ is equal to 0 , while pure mode II is set up when the normal singular stress disappears. $K_{\mathrm{I}_{c}}$ and $K_{\mathrm{II}_{c}}$ can be directly evaluated by Eq. (8) or by the numerical 
calculation method based on the experimental data. Since the interfacial strength of mode II is higher than that of mode $\mathrm{I}, K_{\mathrm{II}_{c}}$ usually possesses the long semi-axis of the elliptical law.

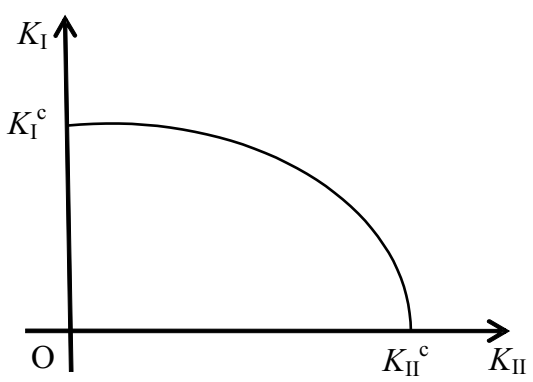

Fig. 2. The mixed - mode interfacial fracture mechanics relating to the stress intensity factor.

An alternative, the mixed-mode interfacial fracture criterion can be expressed by a function of the energy release rate $G$ to the mixed-mode loading phase angle $\psi$

$$
G=\Gamma(\psi)
$$

in which, the mixed-mode loading phase angle $\psi$ is defined as

$$
\psi=\tan ^{-1}\left(\frac{K_{\mathrm{II}}}{K_{\mathrm{I}}}\right)
$$

The interfacial toughness function $\Gamma(\psi)$ is established based on the experimental data and the numerical calculation results. $\Gamma(\psi)$ is an empirical function and has proposed in several forms $[9,10]$. Since the difference of among functions is insignificant, the function below suggested by Kinloch [9] is chosen

$$
\Gamma(\psi)=G_{\mathrm{I}}^{c}\left[1+\tan ^{2}((1-\lambda) \psi)\right]
$$

Fig. 3 illustrates the interfacial toughness function $\Gamma(\psi)$ expressed by Eq. (11).

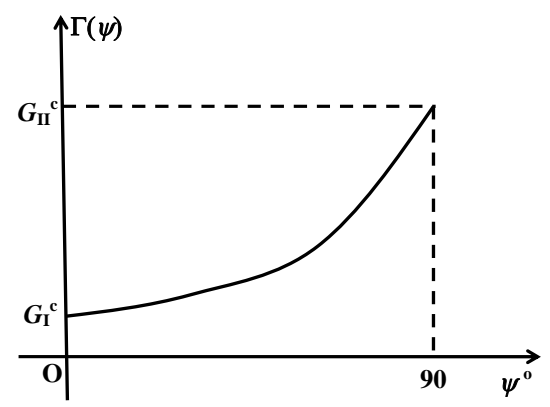

Fig. 3. Interfacial toughness function $\Gamma(\psi)$.

The mix of loading varies from pure mode I to pure mode II with respect to the increment 
of the mixed-mode loading phase angle $\psi$. Pure mode I is found when the total energy release rate reaches a minimum value, and pure mode II is explored when the value of $\psi=90^{\circ}$.

The interfacial fracture criteria are used in the studies, which relate to the interface fracture problems with and without interface crack $[10,11,12]$. The criteria theoretically depend on Dundurs' parameters, and have unique values at a specific mix of loading. It is well known that the second Dundurs' parameter $\beta$ greatly affects both of the energy release rate and the interfacial stress intensity factors, however these effects are usually ignored and lead to the inaccurate criteria in some studies. Moreover, because knowledge about the criteria is not deeply, use of them is not in proper way. In order to clarify the effects of $\beta$ on the criteria, two cases with $\beta=0$ and $\beta \neq 0$ are reviewed in this section.

\subsection{Interfacial fracture criteria with $\beta=0$}

When $\beta=0$ (thus $\varepsilon=0$ by Eq. (3)), the stress intensity factors $K_{I}$ and $K_{I I}$ in Eq. (1) are the same role as their counterparts in elastic fracture mechanics for homogeneous, isotropic solids. $K_{I}$ and $K_{I I}$ are respectively the amplitude of the singularity of the normal and the shear stresses ahead of the crack tip. In addition, the energy release rate $G$ shown in $\mathrm{Eq} .(5)$ is reduced to the origin form in the absence of the mismatch parameter $\beta$. The mixed-mode interfacial fracture criteria finally can be expressed in both of the forms expressed in Eqs. (7) and (11).

\subsection{Interfacial fracture criteria with $\beta \neq 0$}

For bi-materials with $\beta \neq 0$, the normal and the shear stresses on the interface in the dominated zone do not decouple due to the interpenetration of the crack faces and the oscillatory of the singular stresses at some points behind the tip. To define precisely the stress intensity factor in the material systems with $\beta \neq 0$, Eq. (1) is modified as follows

$$
\sigma_{22}+i \sigma_{12}=\left(K_{\mathrm{I}}+i K_{\mathrm{II}}\right)(2 \pi r)^{-1 / 2}\left(\frac{r}{l}\right)^{i \varepsilon}
$$

where $l$ is the reference length. Mode I and II stress intensity factors are defined as decoupling at a distance $l$ ahead of the tip. The proportion of $K_{\mathrm{II}}$ to $K_{\mathrm{I}}$ investigated [10] varies with distance to the tip. The mixed-mode fracture criterion expressed in Eq. (7) is modified as

$$
\left[\left(\frac{K_{\mathrm{I}}}{K_{\mathrm{I} c}}\right)^{2}+\left(\frac{K_{\mathrm{II}}}{K_{\mathrm{II}}}\right)^{2}\right]_{r=l}=1
$$

Similarly, the mixed-mode fracture criterion for an interface crack relating to the energy release rate $G$ with $\beta \neq 0$ is also modified. Rice [13] has proposed the mixed-mode loading phase angle $\psi$ in Eq. (10) as follows

$$
\psi=\tan ^{-1}\left[\left(\frac{K_{\mathrm{II}}}{K_{\mathrm{I}}}\right)_{r=l}\right] \text { or } \psi=\tan ^{-1}\left[\left(\frac{\sigma_{12}}{\sigma_{22}}\right)_{r=l}\right]
$$

where $\sigma_{22}$ and $\sigma_{12}$ are respectively the singular normal and shear stresses near the crack tip. The choice of the reference length $l$ in Eqs. (13) and (14) is arbitrary, but it is usually to choose based on the in-plane length of the specimen geometry, such as a crack length, 
and a material length scale, such as the size of the fracture zone ahead of the crack tip. The modified mixed-mode fracture criterion for the interface crack can be given as

$$
G=\Gamma(\psi, l)
$$

with

$$
\Gamma(\psi, l)=G_{\mathrm{I}}^{c}\left[1+\tan ^{2}((\lambda-1) \psi, l)\right]
$$

Thus the interfacial toughness function depends on not only the mixed-mode loading phase angle $\psi$ but also the reference length $l$. When the reference length changes from $l$ to $l^{\prime}$, the total energy release rate is in the form

$$
\Gamma\left(\psi^{\prime}, l^{\prime}\right)=G_{\mathrm{I}}^{c}\left[1+\tan ^{2}\left((1-\lambda) \psi^{\prime}, l^{\prime}\right)\right]
$$

with

$$
\psi^{\prime}=\psi+\varepsilon \ln \frac{l^{\prime}}{l}
$$

Fig. 4 shows a shift of the toughness function from one choice of reference length to another. According to Eq. (18), when $\varepsilon$ is small enough $\left(\psi^{\prime} \approx \psi\right)$, the change of the reference length does not affect the $\Gamma(\psi, l)$.

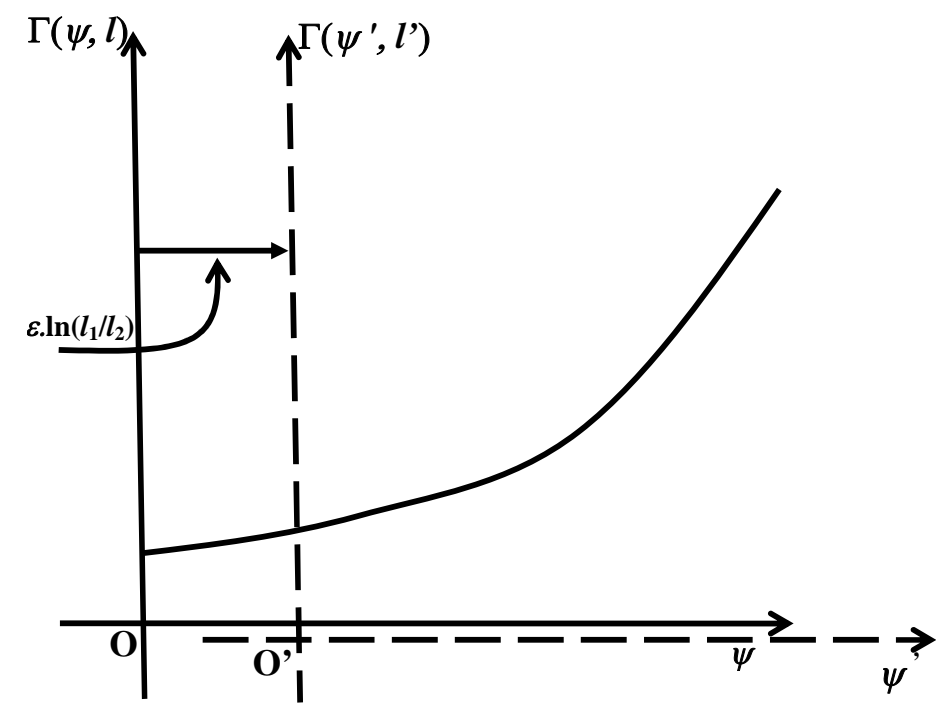

Fig. 4. Effect of the reference length on the toughness function $\Gamma(\psi)$.

Finally, the mixed-mode fracture criteria for interface crack are generally expressed by Eqs. (13) and (16). The choice of the reference length $l$ does not affect the values of $K_{\mathrm{I}_{c}}, K_{\mathrm{II}_{c}}$ and $\Gamma(\psi, l)$ in case $\beta=0$, but has effects on ones in the other cases.

\section{IMPROVED METHOD IN ESTABLISHING THE INTERFACIAL TOUGHNESS FUNCTION}

To clarify the improvements in the proposed methods, the steps in the established process of the interfacial toughness function of two methods are listed in Table 1. 
Table 1. The established processes of the interface toughness function $\Gamma(\psi)$

\begin{tabular}{|c|c|c|}
\hline & The conventional method & The proposed method \\
\hline Step 1 & - preparing the Brazil nut specimens & - Preparing the Brazil nut specimens \\
\hline Step 1-1 & & $\begin{array}{l}\text { - Modeling the specimens, and pre- } \\
\text { determining numerically the placements of } \\
\text { applied load on the specimen where the } \\
\text { mix of loading will occur in pure mode I } \\
\text { and pure mode II. }\end{array}$ \\
\hline Step 2 & $\begin{array}{l}\text { - Doing the experiment at the different } \\
\text { placements of applied load on the speci- } \\
\text { men }\end{array}$ & $\begin{array}{l}\text { - Doing the experiment at two placements } \\
\text { of applied load on the specimen which are } \\
\text { evaluated in step 1-1 }\end{array}$ \\
\hline Step 3 & $\begin{array}{l}\text { - Evaluating } K_{\mathrm{I}_{c}}, K_{\mathrm{II}_{c}}, \psi, G_{\mathrm{I}}^{c} \text { and } G_{\mathrm{II}}^{c} \text { by } \\
\text { the numerical calculation method at the } \\
\text { placements performed in step } 2\end{array}$ & $\begin{array}{l}\text { - Evaluating } K_{\mathrm{I}_{c}}, K_{\mathrm{II}_{c}}, \psi, G_{\mathrm{I}}^{c} \text { and } G_{\mathrm{II}}^{c} \text { by } \\
\text { the numerical calculation method at two } \\
\text { placements performed in step } 2\end{array}$ \\
\hline Step 3-3 & & $\begin{array}{l}\text { - Evaluating the constant } \lambda \text { by Eq. (8) or } \\
\lambda=G_{\mathrm{I}}^{c} / G_{\mathrm{II}}^{c}[10]\end{array}$ \\
\hline Step 4 & $\begin{array}{l}\text { - Establishing the interfacial tough- } \\
\text { ness function } \Gamma(\psi) \text { by the interpolation } \\
\text { method }\end{array}$ & $\begin{array}{l}\text { - Establishing the interfacial toughness } \\
\text { function } \Gamma(\psi) \text { by Eq. (16) }\end{array}$ \\
\hline
\end{tabular}

By comparing between two methods in Table 1, it found that the proposed method needs to add two more steps as Step 1-1 and Step 3-3. However, these additional steps are easily performed by the numerical calculation method, and do not spend so much time. The big differences between two methods are the number of the specimens used in the experiment, and the manner to establish the interfacial toughness function. The conventional method uses many specimens at the different placements of applied load, while the proposed method only uses few specimens at two placements, where pure mode I and pure mode II occur. The interfacial toughness function is established by the interpolation approach in the conventional method, and by an empirical function in the proposed one. Although the number of the specimens used in two methods is quite different, the interfacial toughness functions $\Gamma(\psi)$ obtained are almost in the same form.

\section{CALIBRATION OF THE INTERFACIAL TOUGHNESS FUNCTION $\Gamma(\psi)$}

Two bi-materials prepared and experimented by Wang and Suo [6] were considered in this study. The bi-material of plexiglass/epoxy with $\beta=0$ was mentioned in the first case, and the bi-material of aluminum/epoxy with $\beta \neq 0$ was considered in the second one. The Brazil nut specimens with the radius $a=12.7 \mathrm{~mm}$ and the disk thickness $t=6.35$ $\mathrm{mm}$ as well as the relative crack size $l / a=0.25$ and the epoxy thickness $h=200 \mu \mathrm{m}$ were used in both cases. Fig. 5 shows the typical specimen and loading system. The experiment was conducted at room temperature with an electromechanical Istron machine. The disk deformed linearly until it broken into halves. No plastic deformation was detected in the specimens after the test. 


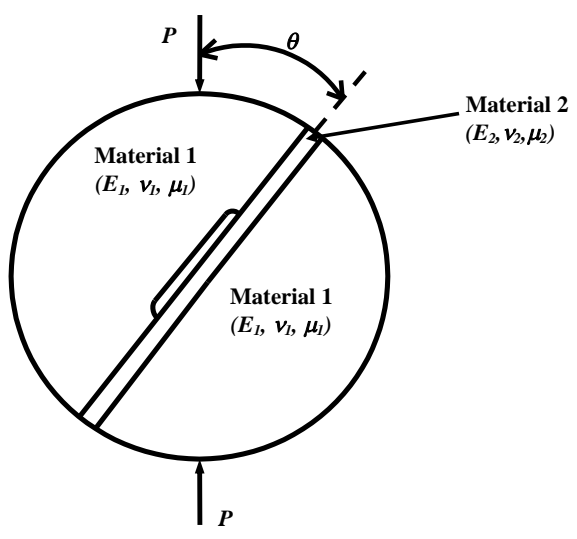

Fig. 5. Specimen and loading system.

\subsection{For bi-material with $\beta=0$}

The elastic constants of the materials of the first case are listed in Table 2. The

Table 2. Material constants of materials in the first case

\begin{tabular}{|l|c|c|}
\hline \multicolumn{1}{|c|}{ Material } & $E(\mathrm{GPa})$ & $\nu$ \\
\hline Plexiglass & 2.4 & 0.38 \\
\hline Epoxy & 1.23 & 0.30 \\
\hline
\end{tabular}

Dundurs' parameter $\beta$ was determined at -0.029 by Eq. (4). Because the magnitude of $\beta$ was small, it was considered as 0 . The error in $G$ in Eq. (5) investigated by Hutchinson and Suo [10] was less than $0.1 \%$. The established process of $\Gamma(\psi)$ as the proposed steps above is performed as follows:

In step 1, the Brazil nut specimens were prepared. Fig. 6 shows the finite element model of a specimen, in which the area near the crack tips of the plexiglass/epoxy interface was carefully divided into the fine elements with the smallest element size of 1 $\mathrm{nm}$. The perfectly bonding condition was assumed on the interfaces. The stresses in the specimens numerically analyzed under plane strain condition by a commercial FEM code. By the preliminary analysis in Step 1-1, pure modes I and II were explored by varying the compression angle $\theta$. Pure mode I was found when the value of $K_{\text {II }}$ was approximately 0 , and pure mode II was established when $K_{\mathrm{I}}$ was eliminated. The values of $\theta$ were determined about $0^{\circ}$ and $25^{\circ}$ corresponding to pure mode I and II, which were similar to those obtained by Wang and Suo [6]

$$
\Gamma(\psi)=31\left[1+\tan ^{2}(0.734 \psi)\right]
$$

In step 2, Wang and Suo [6] used 8 specimens to do the experiment, but the proposed method only used 3 specimens with the compression angles $\theta$ closing to $0^{\circ}$ and $25^{\circ}$, where pure modes I and II occurred. In Step 3, the critical stress intensity factors $K_{\mathrm{I} c}$ at $\theta=0^{\circ}$ and $K_{\mathrm{II}_{c}}$ at $\theta=25^{\circ}$ were respectively determined at $0.22 \mathrm{MPam}^{1 / 2}$ and $0.44 \mathrm{MPam}^{1 / 2}$ by 


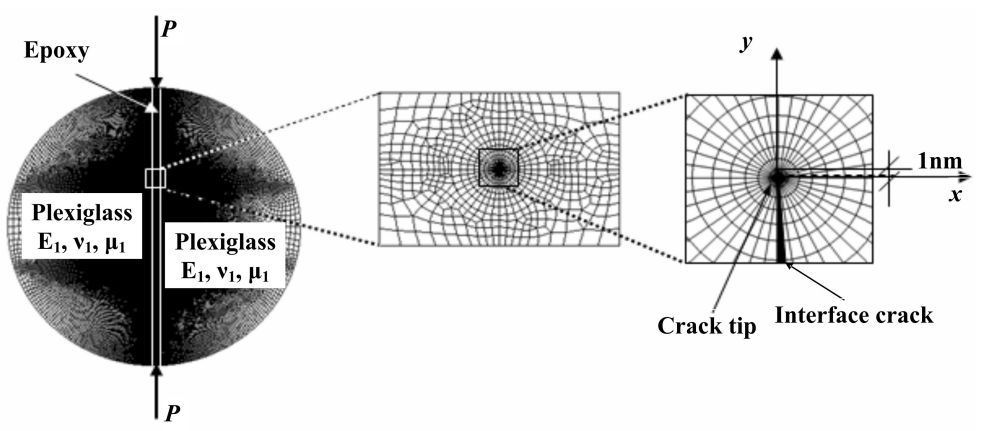

Fig. 6. Finite element model and mesh division near crack tip.

the finite element method. The critical energy release rates $G_{\mathrm{I}}^{c}$ and $G_{\mathrm{II}}^{c}$ evaluated via the J-integral were equal to $32 \mathrm{~J} / \mathrm{m}^{2}$ and $120 \mathrm{~J} / \mathrm{m}^{2}$, respectively. The constant $\lambda$ was evaluated at 0.266 by Eq.(8) (or $\lambda=G_{\mathrm{I}}^{c} / G_{\mathrm{II}}^{c}[10]$ ) in next step. The interfacial toughness function $\Gamma(\psi)$ was finally established by Eq.(16) with an arbitrary reference length $l$.

Fig. 7 shows the interfacial toughness functions $\Gamma(\psi)$ of the interface plexiglass/epoxy with $\beta=0$. The solid line shows the function $\Gamma(\psi)$ obtained by Wang and Suo [6], while the solid line with circle markers illustrates the function $\Gamma(\psi)$ obtained by the proposed method. The functions $\Gamma(\psi)$ are in a close form and the difference between them is less than $5 \%$.

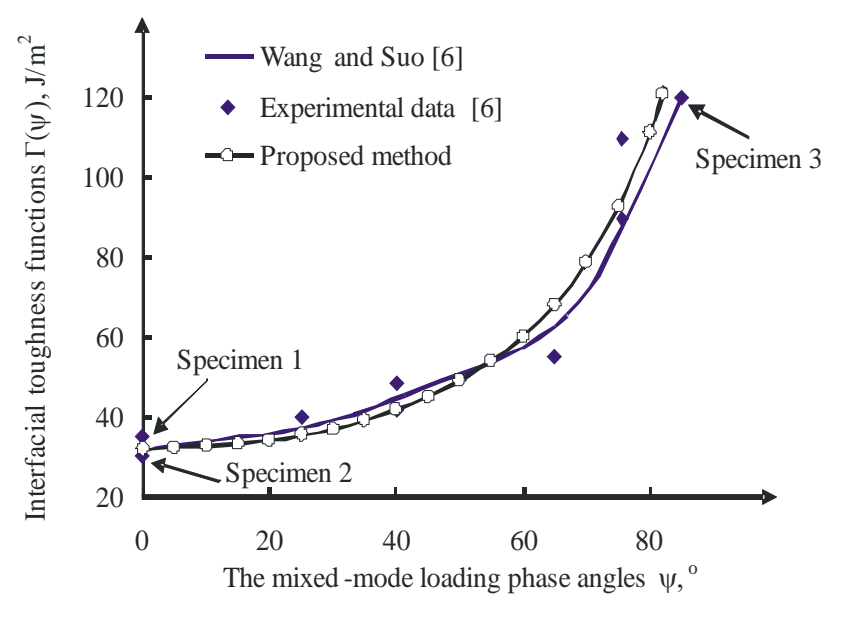

Fig. 7. Interfacial toughness functions $\Gamma(\psi)$ with $\beta=0$.

Thus, the proposed method only used 3 of 8 specimens (numbered in Fig. 7) but the function $\Gamma(\psi)$ was similar to that obtained by the conventional one using 8 specimens. In addition, the interfacial fracture criterion relating to the interfacial stress intensity factors 
can be expressed as

$$
\left(\frac{K_{\mathrm{I}}}{0.22}\right)^{2}+\left(\frac{K_{\mathrm{II}}}{0.44}\right)^{2}=1
$$

where the short semi-axis $K_{\mathrm{I}_{c}}$ and the long semi-axis $K_{\mathrm{II}_{c}}$ are $0.22 \mathrm{MPam}^{1 / 2}$ and 0.44 $\mathrm{MPam}^{1 / 2}$, respectively. The critical stress intensity factor in mode II, $K_{\mathrm{II}_{c}}$, is two times higher than that in mode I, $K_{I c}$. However, based on Eq. (8), the energy release rate $G_{\mathrm{II}}^{c}$ in pure mode II toughness explored is four times higher comparing with $G_{\mathrm{I}}^{c}$ in pure mode I toughness.

\subsection{For bi-material with $\beta \neq 0$}

With the elastic constants of the materials as listed in Table 3, the Dundurs' parameter $\beta$ was determined at 0.218 . Since $\beta \neq 0$, the evaluation of the function $\Gamma(\psi)$ has

Table 3. Material constants of materials in the second case

\begin{tabular}{|l|c|c|}
\hline \multicolumn{1}{|c|}{ Material } & $E(\mathrm{GPa})$ & $\nu$ \\
\hline Aluminum & 70 & 0.35 \\
\hline Epoxy & 1.23 & 0.30 \\
\hline
\end{tabular}

differences comparing with the previous case. The mixed-mode loading phase angle $\psi$ is no longer the independence of the reference length $l$, and is evaluated by Eq. (14). As investigated in Section 2.2, the function $\Gamma(\psi)$ is established similarly to that in case $\beta=$ 0 only when the reference length holds a specific value. With the choice of $l=100 \mu \mathrm{m}$, two unknowns as $G_{\mathrm{I}}^{c}$ and $\lambda$ (or $G_{\mathrm{II}}^{c}$ ) in Eq. (16) need to be determined. It is well known that $G_{\mathrm{I}}^{c}$ and $G_{\mathrm{II}}^{c}$ are respectively the critical energy release rates at pure mode I and pure mode II, and they also correspondingly possesses the minimum and the maximum values in the set of the critical energy release rates. Based on the critical loads at pure modes I and II obtained by the experiment, $G_{\mathrm{I}}^{c}$ and $G_{\mathrm{II}}^{c}$ were respectively determined at $5.5 \mathrm{~J} / \mathrm{m}^{2}$ and $64 \mathrm{~J} / \mathrm{m}^{2}$ via the J-integral. The constant $\lambda\left(=G_{\mathrm{I}}^{c} / G_{\mathrm{II}}^{c}\right)$ was evaluated at 0.086 . The interfacial toughness function $\Gamma(\psi)$ was finally established as follows

$$
\Gamma(\psi)=5.5\left[1+\tan ^{2}(0.914 \psi)\right]_{l=100 \mu \mathrm{m}}
$$

Fig. 8 shows also the interfacial toughness functions $\Gamma(\psi)$ with $l=100 \mu \mathrm{m}$. Because the choice of the reference length $l$ was arbitrary, the critical energy release rate $G_{\mathrm{I}}^{c}$ did not reach the minimum value at $\psi=0^{\circ}$. Thus, the obtained result did not reflect the essence of the mixed-mode fracture behavior. In order to find the reference length satisfying the requirements above, the trial-and-error method was used. By varying the reference length, $l^{\prime}$ was finally determined at $2 \mu \mathrm{m}$, which was about two orders of magnitude smaller. The interfacial toughness function $\Gamma(\psi)$ obtained was established as

$$
\Gamma(\psi)=5.5\left[1+\tan ^{2}(0.914 \psi)\right]_{l=2 \mu \mathrm{m}}
$$

Fig. 8 shows the shift of the $\psi$ - origin by changing from $l$ to $l^{\prime}$. The curve $\Gamma(\psi)$ was close to the experimental data, but had a difference with that obtained by Wang and Suo [6] at the middle values between $G_{\mathrm{I}}^{c}$ and $G_{\mathrm{II}}^{c}$. 


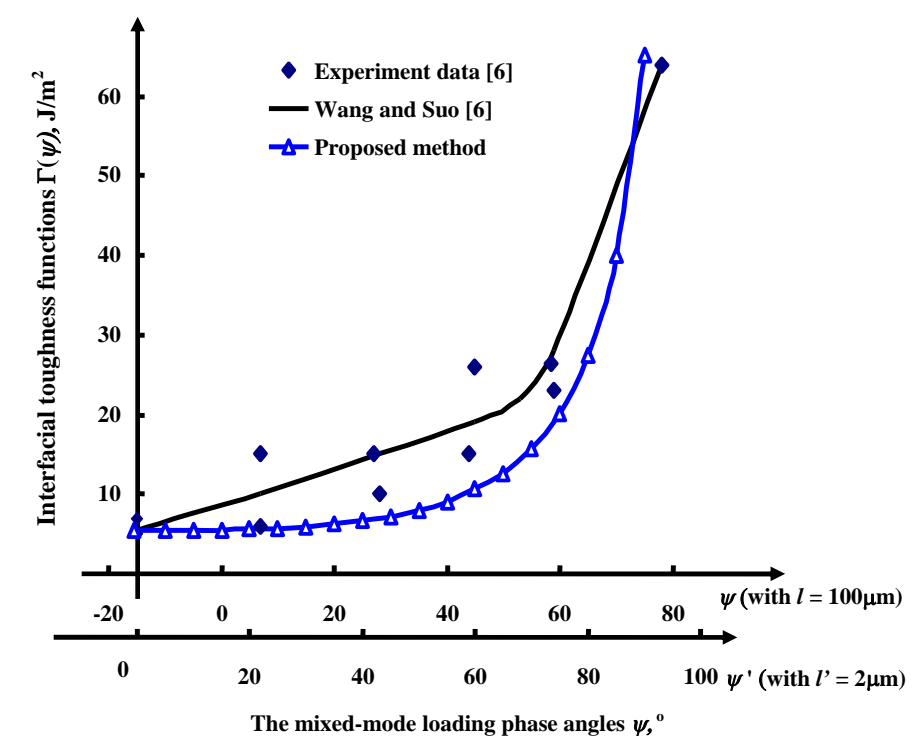

Fig. 8. Interfacial toughness functions $\Gamma(\psi)$ with $\beta \neq 0$.

\section{SUMMARY AND CONCLUSIONS}

In order to establish the interfacial toughness function, a method with two additional steps improved from the conventional method is proposed. In the improved method, a key additional step is the preliminary analysis of the specimen to find the placements of applied load (or the compression angles) where pure modes I and II occur. The experiment only conducted on the specimens at the pre-determined placements of applied load. The critical energy release rates in pure modes I and II were evaluated by the finite element method. The interface toughness function was finally established by an empirical function based on $G_{\mathrm{I}}^{c}$ and $G_{\mathrm{II}}^{c}$. Although the improved method used fewer the specimens than the conventional method, the difference between the interfacial toughness functions was not large. However, in case $\beta \neq 0$, the interface toughness function obtained by the proposed method was close to the experimental data, but had a difference with that obtained by Wang and Suo [6] at the middle values between $G_{\mathrm{I}}^{c}$ and $G_{\mathrm{II}}^{c}$. Moreover, in this paper the mixed-mode interfacial fracture criteria in small-scale yielding condition were reviewed, and the parameters such as the second Dundurs' parameter $\beta$ affecting the criteria were carefully considered as well.

To clarify the improved method, the bi-materials of plexiglass/epoxy with $\beta=0$ and aluminum/epoxy with $\beta \neq 0$ were considered. The experimental data got by Wang and Suo [6] were used. The obtained results could be summarized as follows:

- The constant $\lambda$ considering the influence of the mode II contribution in the criterion was determined at 0.266 for the interface plexiglass/epoxy, and at 0.086 for the interface aluminum/epoxy. Based on $\lambda$, the interface of the bi-material plexiglass/epoxy is known as more brittle than that of the bi-material plexiglass/epoxy. 
- The mixed-mode interfacial fracture criteria of the interface plexiglass/epoxy with $\beta=0$ relating to the interfacial stress intensity factors and the energy release rate were established as (19), (20)

- Based on the trial-and-error method, the reference length $l$ was chosen as $2 \mu \mathrm{m}$ for the interface aluminum/epoxy with $\beta \neq 0$. The criterion of this material system was given as $(22)$

- Although the number of the specimens used in the proposed method was reduced significantly comparing with the conventional one (only 3 of 8 specimens in case $\beta=0$, and only 3 of 10 specimens in case $\beta \neq 0)$, the difference between the function $\Gamma(\psi)$ obtained by the proposed method and the experimental data was slightly.

\section{ACKNOWLEDGMENT}

This paper was supported by Vietnam's National Foundation for Science and Technology Development (NAFOSTED) with No. 107.02.16.09.

\section{REFERENCES}

[1] Y. H. Kim, N. J. Chaug, N. J.Chou, J. Kim, Adhesion of titanium thin film to oxide substrates, Journal of Vacuum Science and Technology A, 5 (1978) 2890 - 2893.

[2] A. J. Kinloch, C. C Lau, J. G Williams, The peeling of flexible laminated, International Journal of Fracture, 66 (1994) 45 - 70.

[3] D. V. Truong, H. Hirakata, T. Kitamura, Effect of loading frequency on fatigue crack growth between a submicron-thick film and a substrate, JSME International Journal A, 49(3) (2006) $370-375$.

[4] T. Kitamura, H. Hirakata, T. Itsuji, Effect of residual stress on delamination from interface edge between nano-films, Engineering Fracture Mechanics , 70 (2003) 2089 - 2101.

[5] S. Z. Y. U Loo, P. C. Lee, Z. X. Lim, N. Yantara, T. Y. Tee, C. M. Tan, Z. Chen, International Journal of Modern Physics B, 24(1\&2) (2010) 164 - 174.

[6] J. S. Wang, Z. Suo, Experimental determination of interfacial toughness curves using Brazilnut sandwiches, Acta Metall. Mater, 38 (1990) 1279 - 1290.

[7] N. Choupani, Interfacial mixed-mode fracture characterization of adhesively bonded joints, International Journal of Adhesion and Adhesives, 28(6) (2008) 267 - 282.

[8] H. M. Jensen, Mixed mode interface fracture criteia, Acta metal. Mater, 38(12) (1990) 2637 $-2644$.

[9] A. J. Kinloch, Adhesion and Adhesives: Science and Technology, Chapman and Hall, London, (1987).

[10] J. W. Hutchinson, Z. Suo, Mixed mode cracking in layered materials, Advances in Applied Mechanics, 29 (1992) 63 - 191.

[11] T. Kitamura, H. Hirakata, D. V. Truong, Initiation of Interface Crack at Free Edge between Thin Films with Weak Stress Singularity, Thin solid Films, 515(5) (2007) 3005 - 3010.

[12] D. V. Truong, T. Kitamura, V. V Thanh, Crack Initiation Strength of an Interface between a Submicron-Thick Film and a Substrate, Materials and Design, 31 (2010) 1450 - 1456.

[13] J. R. Rice, Elastic Fracture Mechanics Concepts for Interfacial Cracks, Journal of Applied Mechanics, 55 (1988) 98- 103. 